Provided for non-commercial research and education use. Not for reproduction, distribution or commercial use.

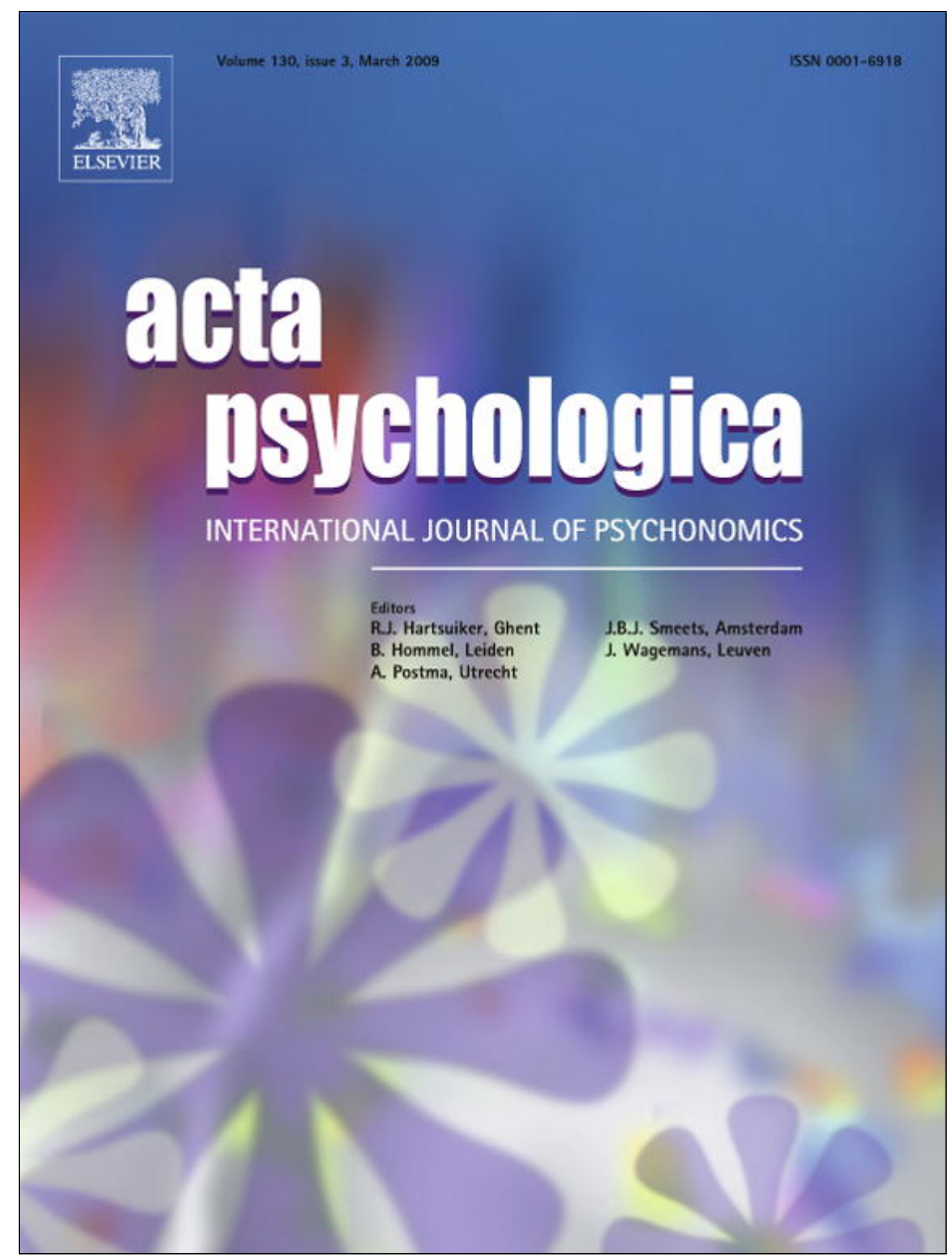

This article appeared in a journal published by Elsevier. The attached copy is furnished to the author for internal non-commercial research and education use, including for instruction at the authors institution and sharing with colleagues.

Other uses, including reproduction and distribution, or selling or licensing copies, or posting to personal, institutional or third party websites are prohibited.

In most cases authors are permitted to post their version of the article (e.g. in Word or Tex form) to their personal website or institutional repository. Authors requiring further information regarding Elsevier's archiving and manuscript policies are encouraged to visit:

http://www.elsevier.com/copyright 


\title{
Haptic mental rotation revisited: multiple reference frame dependence
}

\author{
Robert Volcic $^{\mathrm{a}, \mathrm{b}, *}$, Maarten W.A. Wijntjes ${ }^{\mathrm{a}, \mathrm{c}}$, Astrid M.L. Kappers ${ }^{\mathrm{a}}$ \\ a Helmholtz Institute, Utrecht University, Padualaan 8, 3584 CH Utrecht, The Netherlands \\ ${ }^{\mathrm{b}}$ Psychologisches Institut II, Westfälische Wilhelms-Universität, Fliednerstrasse 21, 48149 Münster, Germany \\ ' Industrial Design Engineering, Delft University of Technology, Landbergstraat 15, 2628 CE Delft, The Netherlands
}

\section{A R T I C L E I N F O}

\section{Article history:}

Received 2 May 2008

Received in revised form 11 December 2008

Accepted 20 January 2009

Available online 24 February 2009

\section{PsycINFO classification:}

2320

Keywords:

Touch

Haptic

Mental rotation

Frame of reference

Space

Hand

\begin{abstract}
A B S T R A C T
The nature of reference frames involved in haptic spatial processing was addressed by means of a haptic mental rotation task. Participants assessed the parity of two objects located in various spatial locations by exploring them with different hand orientations. The resulting response times were fitted with a triangle wave function. Phase shifts were found to depend on the relation between the hands and the objects, and between the objects and the body. We rejected the possibility that a single reference frame drives spatial processing. Instead, we found evidence of multiple interacting reference frames with the hand-centered reference frame playing the dominant role. We propose that a weighted average of the allocentric, the hand-centered and the body-centered reference frames influences the haptic encoding of spatial information. In addition, we showed that previous results can be reinterpreted within the framework of multiple reference frames. This mechanism has proved to be ubiquitously present in haptic spatial processing.
\end{abstract} (c) 2009 Elsevier B.V. All rights reserved.

\section{Introduction}

Any spatial characteristic of an object can only be defined relative to some reference frame, but there are in fact multiple reference frames through which the human system is able to encode objects. For instance, visual information of an object is acquired in retinocentric coordinates, but it can be also encoded in headcentered coordinates to stabilize perception during eye movements, or in body-centered coordinates to allow the perceiver to act on that object. The object can also be encoded relative to the environment in an allocentric reference frame. Similarly, haptic information is usually gathered via the hand, the primary sense organ for touch. The spatial information in hand-centered coordinates is then transposed to hierarchically higher reference frames to fulfill the needs of an active human system. In general, the perceiver's behavior based on both visual and haptic spatial information is assumed to be a result of processes that combine the different frames of reference within each modality as well as between modalities.

Whenever we touch an object we establish a relation between the perceiving hand and the object, and consequently the orienta-

\footnotetext{
* Corresponding author. Address: Psychologisches Institut II, Westfälische Wilhelms-Universität, Fliednerstrasse 21, 48149 Münster, Germany. Tel.: +49 25183 34177.

E-mail address: volcic@psy.uni-muenster.de (R. Volcic).
}

tion of the object with respect to the hand can be obtained from this relation. To extract the spatial characteristics of the object in the environment (i.e., its orientation and its location), additional relations have to be established also between the perceiving hand and the perceiver, and between the perceiver and the surrounding environment. From this point of view, it can be hypothesized that multiple encodings of the same object coexist simultaneously. For instance, Oldfield and Philips (1983) proposed that haptic perception of an object involves both an egocentric frame and an allocentric frame of reference, and that it is the relative position of the egocentric reference frame within the allocentric reference frame that determines the perceptual experience. Similar conclusions were reached also in studies where the task was to identify letters or numbers traced on surfaces of the perceiver's body when the relative spatial orientations and positions of the body surfaces and of the stimuli varied (Corcoran, 1977; Duke, 1966; Krech \& Crutchfield, 1958; Natsoulas \& Dubanoski, 1964; Parsons \& Shimojo, 1987).

The role of reference frames in haptic perception was highlighted in a series of studies investigating the spatial relations between objects (Kappers, 1999; Kappers \& Koenderink, 1999; for a review, see Postma, Zuidhoek, Noordzij, \& Kappers, 2008). Systematic deviations were observed in the task where blindfolded participants were asked to align two objects in such a way that they felt parallel to each other. The two objects had to diverge away from the body, on average by about $50^{\circ}$, to be 
perceived as parallel. A biasing effect of the hand orientation was pinpointed as the dominant factor (Kappers, 2004, 2005; Kappers \& Viergever, 2006; Volcic, Kappers, \& Koenderink, 2007). As a further step, an interaction between the hand-centered egocentric reference frame and the allocentric reference frame was presupposed and subsequently the deviations were successfully described both in two and in three dimensions with a weighted average model that balances the contributions of the two reference frames (Kappers, 2007; Volcic \& Kappers, 2008). These studies have shown the primary role of the hand-centered egocentric reference frame in the encoding of information about objects and how influential this encoding can be in haptic spatial processing.

The interplay of reference frames has also been demonstrated in mental rotation tasks. In vision, for example, different studies have attempted to discover which reference frame is used in a mental rotation task by dissociating the retinal upright from the gravitational upright by having participants tilt their heads in certain conditions (Corballis, Nagourney, Shetzer, \& Stefanatos, 1978; Corballis, Zbrodoff, \& Roldan, 1976). Response times are generally fastest when stimuli are perfectly aligned with the perceptual reference frame. In addition, the degree of misalignment between the orientation of the stimulus and the orientation of the frame produces a linear increase in response time. On the basis of these premises, it is possible to derive in which perceptual reference frame the stimuli are actually encoded. For instance, when the head is tilted, stimuli in gravitational upright orientation are responded to most quickly and response times increase as a function of the misalignment from this orientation. This pattern of response times is consistent with the use of an allocentric, gravitationally aligned, reference frame. On the other hand, a pattern of response times shifted to match the retinally upright orientation is consistent with the use of an egocentric, retinally aligned, reference frame. Corballis et al. $(1976,1978)$ showed that stimuli tend to be encoded in a reference frame midway between the egocentric and the allocentric reference frames, where the latter one is more dominant. McMullen and Jolicoeur (1992) reached similar conclusions.

In a similar fashion, the mental rotation task has been employed to identify the reference frame in which objects are haptically encoded. Carpenter and Eisenberg (1978) presented a single letter haptically in a normal or mirror-image form in various orientations. Participants had to retrieve from memory the letter in its canonical orientation and compare it with the presented letter to decide whether the letter was normal or a mirror image. One of the purposes of their study was to investigate the influence of hand position. They varied the orientation of the hand relative to the participant's body in two conditions while keeping the stimulus in the same location. In the first condition the right hand was parallel to the participant's midsagittal plane, whereas in the second condition the right hand was rotated counterclockwise by $60^{\circ}$. The influence of hand position was evident from the patterns of the response time functions that differed in their phase shift. In both conditions the fastest response time was observed when the hand was aligned with the stimulus, and response times increased with larger differences in orientation between the stimulus and the hand. This means that whereas in the first condition the fastest response time was measured when the major axis of the stimulus was parallel to the participant's midsagittal plane, in the second condition the stimulus had to be rotated by approximately $60^{\circ}$. On the basis of these results, Carpenter and Eisenberg (1978) concluded that the orientation of a letter is encoded with respect to a hand-centered reference frame.

A more recent study on haptic mental rotation led Prather and Sathian (2002) to different conclusions. They applied an embossed letter on the participant's finger pad and, as in Carpenter and Eisenberg (1978), participants had to determine if the letter was normal or a mirror image. In one condition the finger pad was positioned horizontally in front of the participant, centered in the midsagittal plane and parallel to it, whereas in the second condition the finger pad was also centered in the midsagittal plane but orthogonal to it. Despite the change in the orientation of the finger pad and, consequently, in the orientation of the hand, the response time functions in the two conditions were very similar. Prather and Sathian (2002) concluded that haptic stimuli are not encoded in a hand-centered reference frame and suggested that the encoding might occur in a body-, head- or eye-centered reference frame. In addition, they supposed that the phase shift in the direction of the hand orientation found by Carpenter and Eisenberg (1978) could be accounted for by a head-centered reference frame if participants kept their head in alignment with their hand.

The main aim of the present paper was to experimentally disentangle the different reference frames that may play a role in haptic mental rotation and, generally, in haptic spatial processing. To pursue this purpose we used a bimanual mental rotation task that requires participants to determine whether two objects of the same shape and in different orientations felt by the two hands are mirror images of each other or identical except for orientation. This task is also known as the handedness recognition task and it is a widely used task in mental rotation studies since its introduction by Shepard and Metzler (1971). In this way, objects can be directly compared with each other in contrast to the comparison between the stimulus and its memory-based representation as was the case in all the earlier studies on haptic mental rotation. We restricted our quest to a group of reference frames that are most likely involved in haptic spatial processing: the allocentric, the hand-centered egocentric and the body-centered egocentric reference frames. In the allocentric reference frame, objects are represented relative to the environment that is extrinsic to the perceiver. In the hand-centered egocentric reference frame, objects are represented relative to the perceiver's hand and, finally, in the body-centered egocentric reference frame, objects are represented relative to the perceiver's body. Our definition of the latter reference frame comprises also a head-centered reference frame as long as the head faces forward. To dissociate the influences of the different reference frames, we devised different experimental conditions in which the two objects to be compared were explored with different hand orientations and were located in different positions relative to the perceiver's body (see Fig. 1, left panel). We expected that the employment of the relevant reference frame would evince itself in a specific phase shift of the response time function. In the simplest case, if only an allocentric reference played a role, the quickest response time should be observed when the two objects are physically aligned. Response times would linearly increase, both in the positive and in the negative directions, as a function of an increase in the orientation difference between the two objects. This pattern, i.e., a response time function with no phase shift, would be independent of the experimental condition and it is exemplified in the leftmost column in Fig. 1 (right panel). This is essentially similar to the Shepard and Metzler (1971) model. They used a linear function to model response times. The triangle wave function is the generalized version of this function taking into account the periodicity. Predictions that are dependent on the experimental condition could be made for the cases in which either the hand-centered or the body-centered reference frame would play a role in haptic mental rotation. The response time function was expected to shift horizontally (phase shift) depending on the positions of the hands or on the position of the objects with respect to the body (see center and rightmost columns in Fig. 1, right panel). For example, if the body-centered 

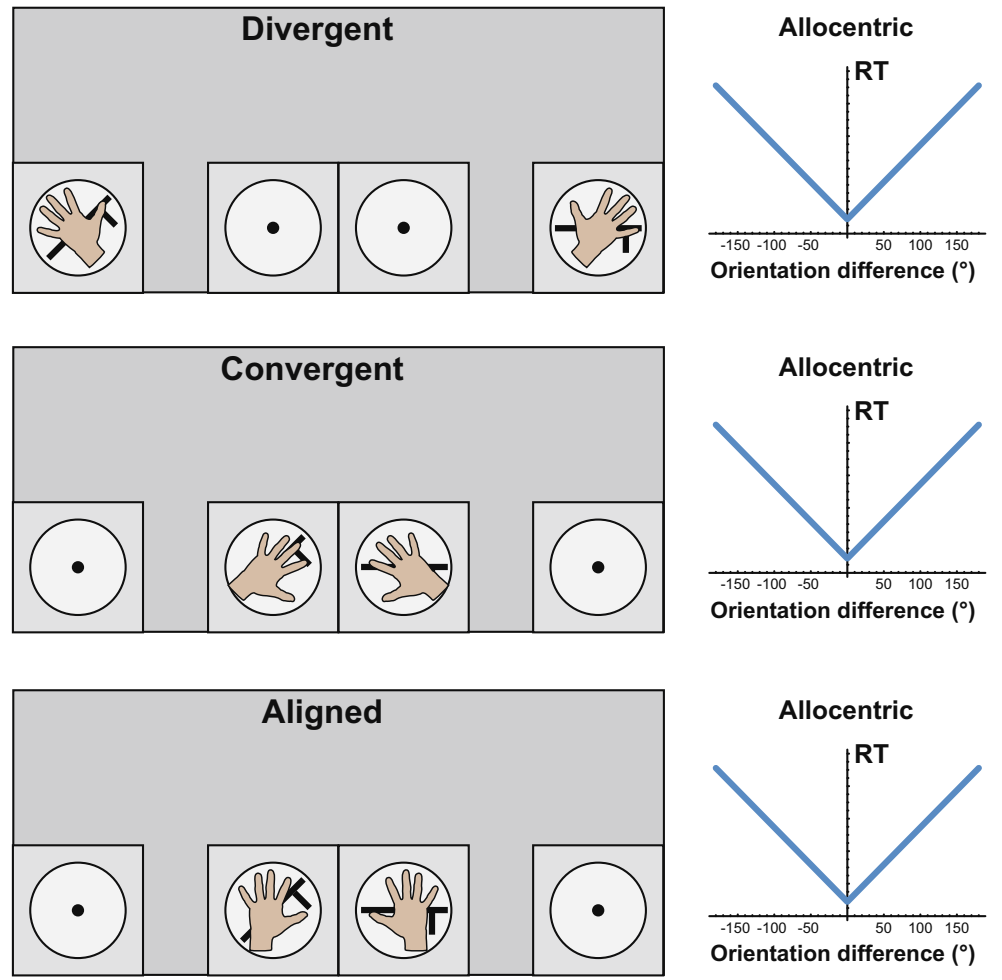
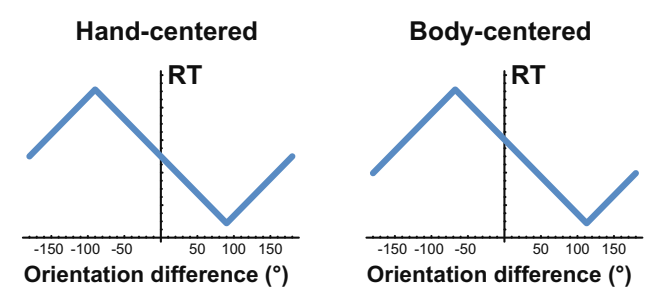

Hand-centered
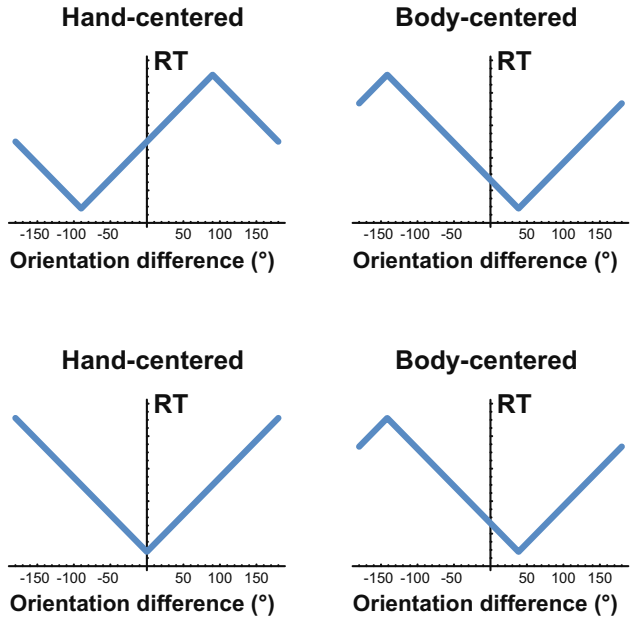

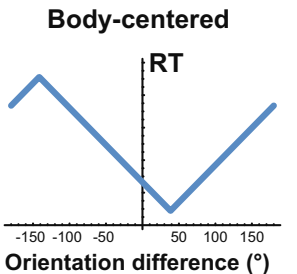

Fig. 1. Experimental conditions (left panel) and predictions of the response time (RT) function for each condition according to the allocentric, the hand-centered and the body-centered reference frames (right panel). Participants could freely explore the objects during the experiment with the only constraint being the orientation of the hands determined by the condition.

reference frame is used, the parity of objects oriented similarly with respect to the radial direction of the body midline should be identified fastest. The direction and the magnitude of these phase shifts would therefore indicate the perceptual reference frame in which the objects are encoded. These predictions were based on a selective process, in which only one reference frame is selected and guides haptic spatial processing without any influence from other reference frames. Contrary to this view, recent studies mentioned earlier have shown that the haptic perception of spatial relations is based on a combination of multiple frames of reference. In line with this interpretation, we expected interacting reference frames also in haptic mental rotation. As a corollary, any phase shift intermediate to the predictions made on the basis of the selective process would suggest a mechanism that combines the different reference frames. In this case, we expected each reference frame to contribute to haptic spatial processing according to a specific weight. An interesting aspect was also to compare the contributions of the different reference frames with those found in the previous studies on haptic perception of spatial relations. For instance, the large contribution of the allocentric reference frame in the parallelity task might be inherent to the task, since the concept of parallelity is defined with respect to this reference frame. On the other hand, in the mental rotation task the choice of which reference frame to use is in principle arbitrary. The haptic mental rotation task thus reveals in which combination of reference frames objects are encoded in a recognition task, instead of a manipulation task. The second difference between the two tasks regards the temporal execution. In the mental rotation task participants are asked to respond as fast as possible, whereas in the parallelity task the available time to perform the task is less stringent. Presumably, these differences might have an impact on the relative contributions of the different reference frames.

\section{Method}

\subsection{Participants}

Eight male participants took part in this experiment. Participants M.W. and R.V. are authors of the paper. All the others were undergraduate students and were remunerated for their efforts. Participants had normal haptic, somatosensory and motor functioning. None of the participants (except the authors) had any prior knowledge of the experimental design and the task.

\subsection{Apparatus and stimuli}

The set-up consisted of a table $(150 \times 75 \times 75 \mathrm{~cm})$ on which two iron plates $(30 \times 30 \mathrm{~cm})$ could be positioned, one on either side of the participant's midsagittal plane. The iron plates were covered with a plastic layer on which a protractor with a radius of $10 \mathrm{~cm}$ was printed. The centers of the two protractors were either 30 or $120 \mathrm{~cm}$ apart and $15 \mathrm{~cm}$ from the long table edge. The participants were seated on a stool in a fixed position in front of the table. Their body midline was $15 \mathrm{~cm}$ from the edge of the table. The participant's head faced forward during experimental sessions. An aluminum object, with an axle in the middle, was inserted in the center of each protractor and could be rotated freely by the experimenter. The objects used as stimuli were made of two cylindrical bars, with a diameter of $1 \mathrm{~cm}$ (see Fig. 2). The main bar had a length of $20 \mathrm{~cm}$, and attached to this at $5 \mathrm{~cm}$ from the center was a smaller bar with a length of $5 \mathrm{~cm}$. One pair of objects had the smaller bar attached on the right side of the main bar, whereas the other pair had it attached on the left side. The main bar had arrowshaped ends that allowed the orientation to be read off with an accuracy of $0.5^{\circ}$. Small magnets were attached under the bar to prevent accidental rotations. 

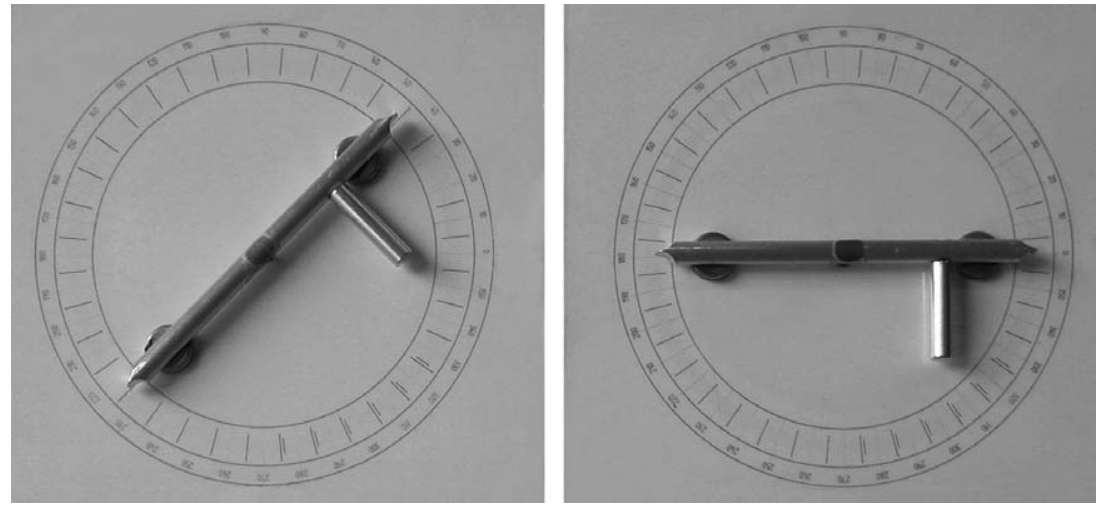

Fig. 2. A top view of a pair of stimuli positioned on their protractors. In this instance, the two stimuli are identical except for their orientation.

The objects were connected to electrical wires, which were plugged into the touch sensitive contact of the custom build stand-alone response time measuring device. The moment the hands touched the stimuli, the contact was registered through a touch sensitive contact and response time measurement started. Inside the device, a $50 \mathrm{~Hz}$ electrical field was generated. When the objects were touched the impedance change caused a drop in the field frequency. If this change reached a certain threshold the time measurement started. Time measurement terminated with a vocal response registered through a headset microphone that was also attached to the measuring device. The response time was then fed into a computer, where it was stored. The software that communicated with the device was written in LabView ${ }^{\mathrm{TM}}$.

Stimuli were presented in pairs. The stimulus on the left side of the participant's midsagittal plane was oriented at $45^{\circ}, 135^{\circ}, 225^{\circ}$, or $315^{\circ}$. The $0^{\circ}$ orientation was aligned along the left-right axis of the table and an increase in degrees signifies a rotation in the counterclockwise direction. The stimulus on the right side was rotated with respect to the left stimulus for a number of degrees between $-170^{\circ}$ and $170^{\circ}$ in steps of $20^{\circ}$. Each stimulus was paired with either another identical stimulus (Same trial) or its mirror version (Different trial). The stimuli were presented in three different experimental conditions. In the Divergent condition, the two stimuli were located $120 \mathrm{~cm}$ apart and when touching them the two hands were diverged by approximately $90^{\circ}$ (see Fig. 1, left panel, top). In the Convergent condition, the two stimuli were $30 \mathrm{~cm}$ apart and when touching them the two hands were converged by approximately $90^{\circ}$ (see Fig. 1, left panel, middle). In the Aligned condition, the stimuli were again $30 \mathrm{~cm}$ apart and the two hands were aligned with each other (see Fig. 1, left panel, bottom). In total, each participant completed 864 trials ( 2 objects $\times 4$ orientations of the left located object $\times 18$ orientations of the right located object $\times 2$ same/different pairs $\times 3$ conditions). The order of trials in each experimental condition was random and different for each participant. The order of the experimental conditions was counterbalanced across participants.

\subsection{Procedure}

Participants had to perform a haptic mental rotation task. The experimenter set two stimuli in their locations and gave a start signal to the participant. The blindfolded participants were instructed to touch the two objects simultaneously with their hands oriented in the way predefined by the condition. They had to respond as fast as possible whether the two stimuli were the same or different. It was also emphasized that the answer should be correct. Participants received feedback on whether their answer was correct. When an incorrect response was given, the trial was repeated at the end of the experimental condition so that in the end a full set of correct trials was measured. The response time measurement initiated when the participant first touched the stimuli and terminated when the participant verbally responded either "same" or "different". In between the trials, participants rested their hands on the surface of the table.

The experimental conditions were preceded by practice trials until the participant was confident with the execution of the task. For none of the participants did the training session exceed 80 trials. The word "rotation" was not used in the instructions.

The experimental sessions ended after $1 \mathrm{~h}$ to prevent fatigue of the participants and were performed on separate days. Therefore, each of the three experimental conditions spanned over multiple sessions, but each of them was always completed before the start of the next one. Participants took on average $8 \mathrm{~h}$ to complete all conditions.

\subsection{Data analysis}

The error rates in all the experimental conditions were low (below 5\%) and were not further analyzed. Data analysis of the response times was limited to the Same trials, because the Different trials do not convey any information since the angle through which different objects must be rotated to achieve congruence is not defined.

\subsubsection{Fitting procedure}

The individual response times of the Same trials were grouped for each condition and orientation difference. Since the response times are usually not normally distributed, we took for each orientation difference the median response time of the grouped data. These medians were then used to fit a triangle wave function through the data of each participant (see Fig. 3). The triangle wave

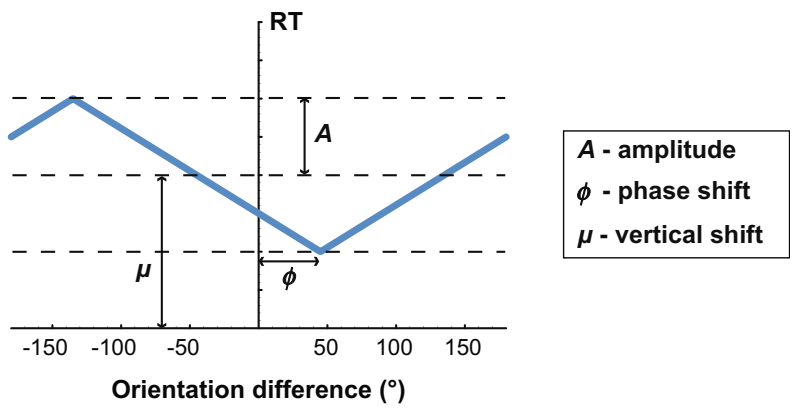

Fig. 3. Triangle wave function used to fit the response times as a function of the orientation difference between the two objects. The function is defined by three parameters: amplitude $(A)$, phase shift $(\phi)$ and vertical shift $(\mu)$. RT is response time. 
function is a periodic function with a fixed wave period of $360^{\circ}$ and is expressed as

$T(x, A, \phi, \mu)=2 A\left|\operatorname{Int}\left(\frac{x-\phi}{360^{\circ}}\right)-\frac{x-\phi}{360^{\circ}}\right|+\mu-\frac{A}{2}$,

where $A$ is the amplitude, $\phi$ is the phase shift and $\mu$ is the vertical shift. The function $\operatorname{Int}(x)$ gives the integer closest to $x$. For $\phi=0$, the function is essentially identical to the function used by Shepard and Metzler (1971). Note that the triangle wave function is not continuously differentiable and can thus not describe a physical phenomenon. This issue, though, is of minor concern for the purposes of this study.

\subsubsection{Estimation of the weighting factors}

We suppose that the phase shift of the response time function is determined by a weighted contribution of an allocentric reference frame, a hand-centered egocentric reference frame and a bodycentered egocentric reference frame. Therefore, the phase shift of the response time function can be expressed by the following equation:

$$
\begin{gathered}
\phi=\left(1-w_{1}-w_{2}\right) \phi_{\text {Allo }}+w_{1} \phi_{\text {Hand }}+w_{2} \phi_{\text {Body }} \\
\left(0 \leqslant w_{1} \leqslant 1,0 \leqslant w_{2} \leqslant 1\right),
\end{gathered}
$$

where $\phi_{\text {Allo }}, \phi_{\text {Hand }}$ and $\phi_{\text {Body }}$ correspond, respectively, to the phase shifts that would be expected if only an allocentric, a hand-centered egocentric or a body-centered egocentric reference frame was used in a specific condition. These values are fixed and are defined on the basis of the position of the participant with respect to the set-up. The size of the weighting factors $\left(w_{1}, w_{2}\right)$ modulates the relative contributions of the three reference frames. The weighting factors are the parameters used in the fitting procedure.

The above equation can be applied to each of the three experimental conditions of our study. Since we assume that the weighting factors are independent from the location in space, our intent was to compute weighting factors that could capture the phase shifts of all three conditions. Hence, to estimate the weighting factors, that is, the contributions of the different reference frames, we defined this set of equations:

$$
\left\{\begin{array}{l}
\phi_{\text {Div }}=\left(1-w_{1}-w_{2}\right) \phi_{\text {Allo_Div }}+w_{1} \phi_{\text {Hand_Div }}+w_{2} \phi_{\text {Body_Div }} \\
\phi_{\text {Conv }}=\left(1-w_{1}-w_{2}\right) \phi_{\text {Allo_Conv }}+w_{1} \phi_{\text {Hand_Conv }}+w_{2} \phi_{\text {Body_Conv }} \\
\phi_{\text {Alig }}=\left(1-w_{1}-w_{2}\right) \phi_{\text {Allo_Alig }}+w_{1} \phi_{\text {Hand_Alig }}+w_{2} \phi_{\text {Body_Alig }}
\end{array}\right.
$$

and extracted the weighting factors through a least-square error minimization procedure. The estimation of the weighting factors was performed individually per participant, because participantdependent differences in the weights might be expected. The terms $\phi_{\text {Div }}, \phi_{\text {Conv }}$ and $\phi_{\text {Alig }}$ correspond, respectively, to the phase shifts measured in the three experimental conditions. All the other $\phi$ terms correspond to the predicted phase shifts that depend on the experimental condition and are represented in Fig. 1 (right panel). The predicted phase shifts were calculated on the basis of the position and orientation of the objects with respect to the orientations of the hands for the hand-centered reference frame predictions, and with respect to the body midline for the body-centered reference frame predictions. For instance, two objects are defined as having the same orientation within a certain reference frame when they are identically oriented with respect to either the hand or the imaginary lines irradiating from the participant's body midline. The predictions for the allocentric reference frame are independent of the position of the objects with respect to each other or with respect to the participant and are therefore constant.

\section{Results}

The graphs in Fig. 4 show the response times as a function of the orientation difference between the two objects averaged over all participants in the Divergent, Convergent and Aligned conditions. The triangle wave function was fitted through the data. Several observations can be made already at first sight. First of all, the phase shift of the fitted function relative to the physical alignment varies in the different conditions and can largely deviate from zero. This indicates that the lowest response times were measured when the two objects actually had a different orientation and were not physically aligned with each other. Second, from the point defined by the phase shift, the response times showed a positive linear relationship with respect to smaller and larger orientation differences supporting the adoption of the triangle wave function as the fitting function. The explained variance was between $85 \%$ and $93 \%$.

In the Divergent, Convergent and Aligned conditions, the phase shifts of the response time function were $91.1^{\circ},-23.8^{\circ}$, and $8.9^{\circ}$, respectively. Note that the direction of the phase shifts corresponds with the relative orientations of the two hands touching the objects. For instance, in the Divergent condition the lowest response times were measured when the two objects were approximately orthogonal to each other, but actually had very similar orientations with respect to the left and right hands. In the Convergent condition the direction was in agreement with the relative orientation of the hands, although the magnitude of the phase shift was reduced. The phase shift in the Aligned condition was still positive, but close to the actual physical alignment of the objects.

To further analyze the response time measurements we looked at the data of the individual participants. Table 1 charts the parameters of the fitting function for each condition and each participant. In addition, the $R^{2}$ of each fit are listed in the rightmost column showing good fits for all participants and conditions. Three repeated measures ANOVAs were performed on the amplitude $(A)$, on the phase shift $(\phi)$ and on the vertical shift $(\mu)$ with the experimental conditions as a factor. Both the amplitudes and the vertical shifts were not significantly different among the three experimental conditions. Therefore, the average response time and the range of the response times were stable in all the conditions. Consequently, also the speed of rotation $\left(180^{\circ} /(2 A)\right)$ was fairly invariable: $195.9 \% \mathrm{~s}, 206.1^{\circ} \mathrm{s}$, and $184.1 \%$, in the Divergent, Convergent and Aligned conditions, respectively. On the other hand, the repeated measures ANOVA on the phase shifts revealed a significant effect of condition $(F(2,14)=180.54, p<.0001)$. Subsequent pairwise comparisons with Bonferroni corrections showed significant differences between the Divergent and Convergent conditions $(p<.0001)$, between the Divergent and Aligned conditions $(p<.0001)$, and between the Convergent and Aligned conditions $(p<.005)$. The phase shifts of each participant are represented in the bar chart shown in Fig. 5. The phase shifts agree quite well across participants. ${ }^{1}$

The phase shifts do not match perfectly with any of the predicted phase shifts (see Fig. 1, right panel). The use of an allocentric reference frame does not predict any phase shift. The use of a hand-centered egocentric reference frame predicts a larger negative phase shift in the Convergent condition. And, finally, the use of a body-centered egocentric reference frame predicts a positive

\footnotetext{
${ }^{1}$ The same results were obtained by excluding from the analyses the data sets of the two authors: both the amplitudes and the vertical shifts were not significantly different among the three conditions and the phase shifts differed significantly $(F(2,10)=106.897, p<.0001)$. Significant differences were found in the pairwise comparisons with Bonferroni corrections between the Divergent and the Convergent conditions $(p<.0001)$, between the Divergent and Aligned conditions $(p<.0001)$, and between the Convergent and Aligned conditions $(p<.05)$.
} 

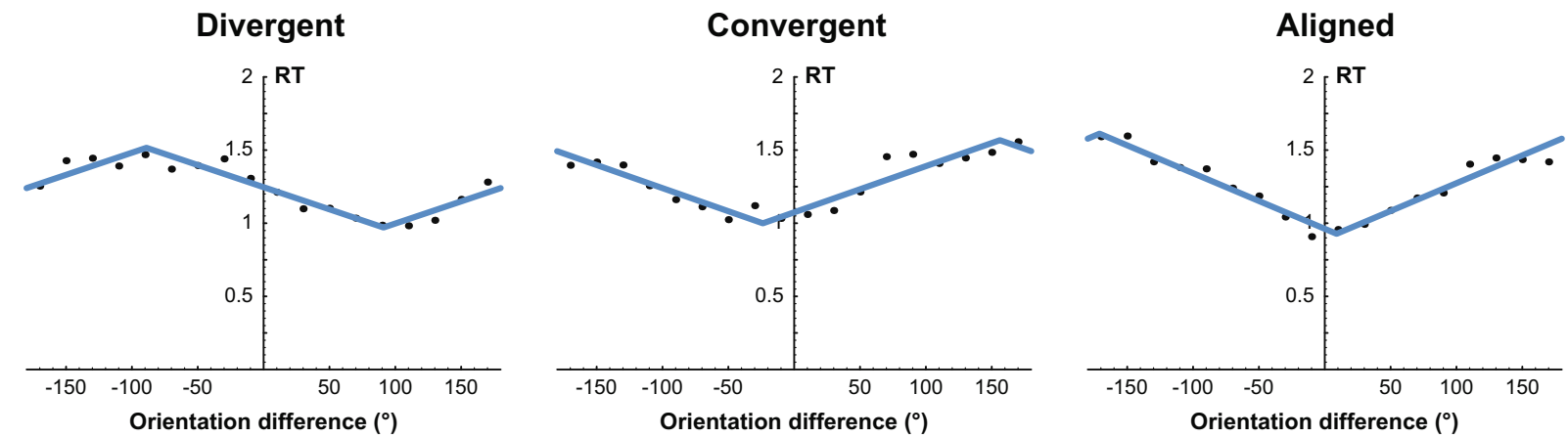

Fig. 4. Response times as a function of the orientation difference averaged over all participants for the Divergent, Convergent and Aligned conditions. Data are fitted by the triangle wave function.

Table 1

For every participant and every condition, their amplitude $(A)$, phase shift $(\phi)$, vertical shift $(\mu)$ and $R^{2}$ of the triangle wave function fit. For every condition and every parameter mean values and standard deviations $(S D)$ are shown.

\begin{tabular}{|c|c|c|c|c|c|}
\hline Condition & Participant & $A(\mathrm{~s})$ & $\phi\left(^{\circ}\right)$ & $\mu(\mathrm{s})$ & $R^{2}$ \\
\hline \multirow[t]{10}{*}{ Divergent } & M.W. & 0.87 & 90.3 & 1.13 & .89 \\
\hline & R.V. & 0.48 & 94.5 & 0.96 & .57 \\
\hline & B.K. & 0.68 & 85.1 & 1.22 & .70 \\
\hline & D.B. & 0.40 & 92.9 & 1.05 & .67 \\
\hline & O.D. & 0.94 & 97.3 & 1.53 & .63 \\
\hline & К.B. & 0.28 & 88.9 & 0.98 & .44 \\
\hline & B.L. & 0.47 & 73.0 & 1.66 & .42 \\
\hline & J.W. & 0.29 & 91.5 & 1.41 & .73 \\
\hline & Mean & 0.55 & 89.2 & 1.24 & .63 \\
\hline & $S D$ & 0.25 & 7.5 & 0.26 & .15 \\
\hline \multirow[t]{10}{*}{ Convergent } & M.W. & 0.91 & -40.6 & 1.05 & .86 \\
\hline & R.V. & 0.62 & -16.4 & 0.92 & .89 \\
\hline & B.K. & 1.35 & -39.3 & 1.48 & .69 \\
\hline & D.B. & 0.26 & -64.2 & 1.01 & .51 \\
\hline & O.D. & 0.99 & 4.4 & 1.24 & .82 \\
\hline & K.B. & 0.33 & -23.4 & 0.81 & .55 \\
\hline & B.L. & 0.22 & -48.4 & 1.71 & .64 \\
\hline & J.W. & 0.41 & -6.3 & 2.05 & .47 \\
\hline & Mean & 0.64 & -29.3 & 1.28 & .68 \\
\hline & $S D$ & 0.41 & 22.9 & 0.43 & .16 \\
\hline \multirow[t]{10}{*}{ Aligned } & M.W. & 0.63 & 9.2 & 0.91 & .85 \\
\hline & R.V. & 0.71 & 4.4 & 0.93 & .81 \\
\hline & B.K. & 0.73 & 16.6 & 1.08 & .65 \\
\hline & D.B. & 0.50 & 9.6 & 1.19 & .43 \\
\hline & O.D. & 0.98 & 23.6 & 1.56 & .74 \\
\hline & K.B. & 0.26 & 19.1 & 0.88 & .57 \\
\hline & B.L. & 0.38 & 0.1 & 1.49 & .34 \\
\hline & J.W. & 0.40 & 0.9 & 2.11 & .74 \\
\hline & Mean & 0.57 & 10.4 & 1.27 & .64 \\
\hline & $S D$ & 0.23 & 8.6 & 0.43 & .18 \\
\hline
\end{tabular}

phase shift in the Convergent condition. Our next step was therefore to investigate if the measured phase shifts can be explained by a combination of the three reference frames. The question was if a combination of weighting factors exists that integrates the contributions of the reference frames in such a way as to be consonant with the measured phase shifts. The error minimization procedure explained in the Methods section was applied to estimate the weighting factors for each participant separately. The resulting weights are shown in Fig. 6. It is evident that the handcentered egocentric reference frame plays the primary role. The body-centered egocentric reference frame appeared to be the second most influential reference frame; finally, the allocentric reference frame was only minimally involved. On average, the weights were $.15, .60$, and .25 for the allocentric, the hand-centered egocentric and the body-centered egocentric reference frames. In addition, it is interesting to observe that since the contribution of the allocentric reference frame is uniform across participants $(S D=.069)$, participants differ among each other only with respect to the contributions of the hand- and body-centered reference frames $(S D=.132$ and $S D=.108$, respectively).

Given that the contribution of the allocentric reference frame was small, we evaluated both a model with all three reference frames and a model without the allocentric reference frame. The two models were compared on the basis of a standard procedure for model selection - the corrected Akaike information criterion $\left(\mathrm{AIC}_{\mathrm{C}}\right.$ ), which evaluates the complexity of the model (number of parameters) against its accuracy in fitting the data (e.g., Wagenmakers \& Farrell, 2004). This method allows the comparison of the Akaike weights, that is, the relative probabilities of each model being correct among the candidate models. For all participants, the model with the three reference frames proved to be better than the other model with an average probability of .78 .

\section{Discussion}

In the present study, we investigated the role that reference frames play in haptic spatial processing. In a bimanual haptic mental rotation task, participants had to compare the parity of two objects located in different spatial locations by exploring them with different hand positions. Our goal was to dissociate the influence of the allocentric, the hand-centered egocentric and the body-centered egocentric reference frames. The idea that only one of these reference frames is selected and drives haptic spatial processing was rejected. Instead, our data support a dependence on a combination of multiple reference frames, in which the hand-centered reference frame plays the central role.

The influence of a specific reference frame was expected to act on the phase shift of the response time function. If a single reference frame among the allocentric, the hand-centered and the body-centered reference frames had been involved, we would observe the phase shifts represented in Fig. 1 (right panel). In fact, the patterns of the response time function were different. The phase shift was modulated by the diverse hand postures and positions of the objects with respect to the perceiver. However, the causing factor cannot be attributed to the influence of a single reference frame. A dominant role of the allocentric reference frame was excluded merely on the fact that phase shifts were observed. A prevailing role of the body-centered reference frame was also excluded, since it could never have predicted a negative phase shift in the Convergent condition. On the other hand, hand-centered reference frame predictions were successful in capturing the directionality of the shifting response time function, although also in this case the observed phase shift diverged from the predicted ones. On the basis of these observations we discarded any explanation that involves an exclusive use of a particular reference frame, 


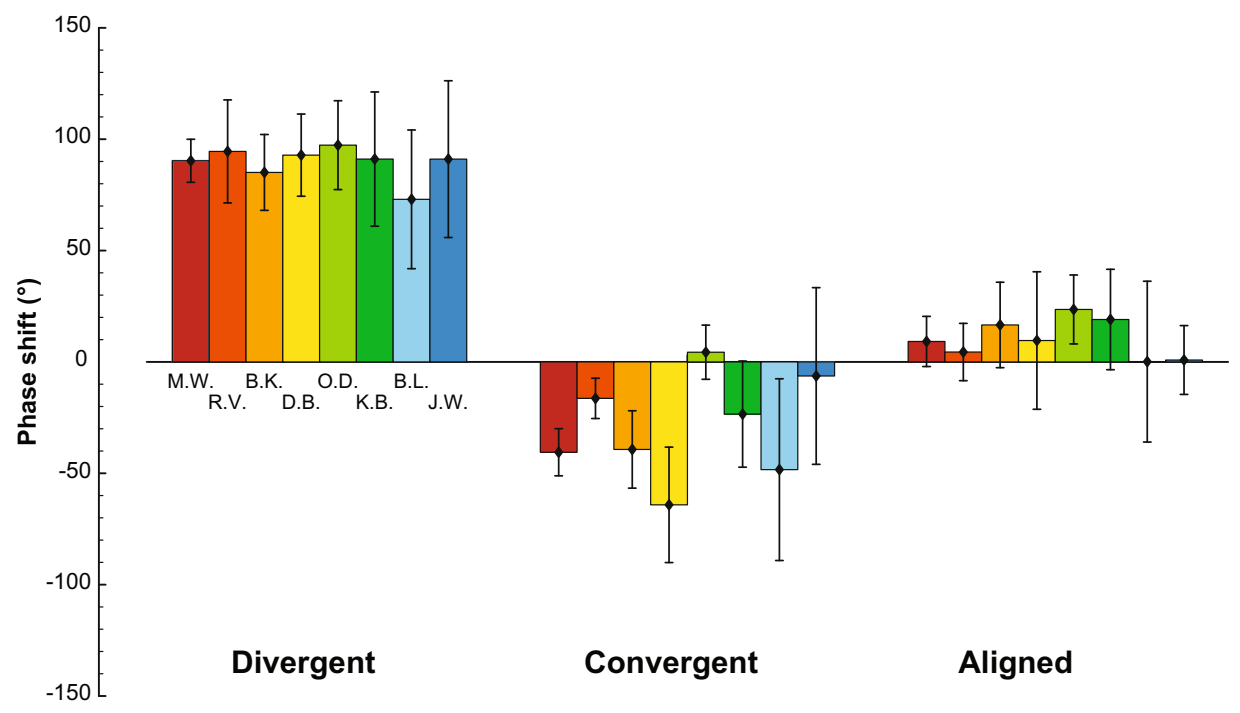

Fig. 5. Phase shifts $(\phi)$ of each participant in the Divergent, Convergent and Aligned conditions. Error bars indicate the $95 \%$ confidence interval of the standard error of the mean as obtained from the triangle wave function fits.

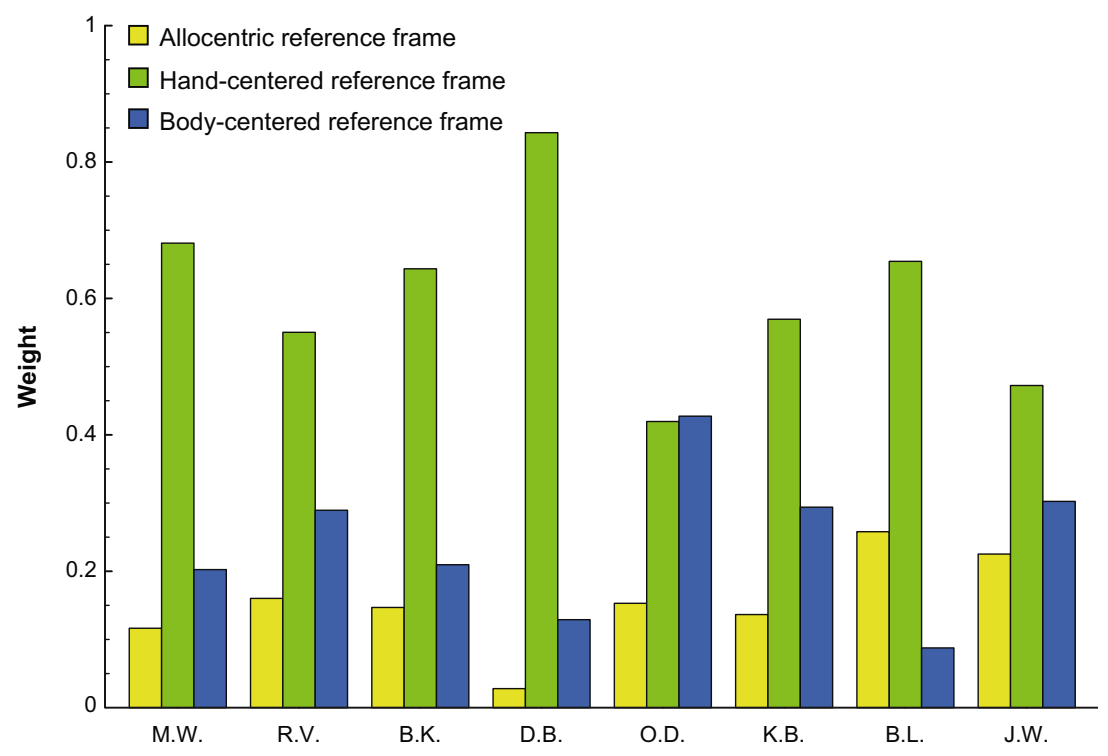

Fig. 6. Estimated weights of the allocentric, the hand-centered and the body-centered reference frames for each participant.

in contrast to Carpenter and Eisenberg (1978) and Prather and Sathian (2002). Rather, we interpreted the phase shifts of the response time function as a product of the interplay of the allocentric, the hand-centered and the body-centered reference frames. Each of these reference frames actively contributed to the processing of spatial information, but each of them to a different extent, which was estimated by computing the weighting factors. The hand-centered reference frame predominated as the most influencing factor, with the body-centered reference frame placed second, and the allocentric reference frame showing the smallest contribution. This combination of reference frames with their specific weights could actually account for the directions and the extents of the phase shifts in our experimental conditions. None of these reference frames could be excluded without noticeably affecting the accuracy.

In the previous studies on haptic mental rotation, it was suggested that a predominant hand-centered reference frame accounted for the results of Carpenter and Eisenberg (1978), but not for the results of Prather and Sathian (2002). In order to fairly compare past and present results, we extracted the response time data from the above-mentioned studies and fitted the triangle wave function to estimate the phase shifts. In Carpenter and Eisenberg (1978), we found a phase shift of $48^{\circ}$ in the direction of the hand-centered reference frame; and in Prather and Sathian (2002), we found a phase shift of $29^{\circ}$. In the first study, a $60^{\circ}$ phase shift would support the view of a complete hand reference frame dominance, whereas in the second study, the phase shift should have been of $90^{\circ}$. The prediction for the body-centered reference frame in Carpenter's study could not be clearly defined, since the exact location of the stimulus with respect to the body is not known. In Prather's study, on the other hand, the body-centered reference frame does not predict any phase shift. Importantly, we confirmed the role of the hand-centered reference frame in Carpenter and Eisenberg (1978), but we also found evidence of the influence of the hand-centered reference frame in Prather and Sathian (2002). This evidence contrasts with their conclusions, 
which excluded any role of this reference frame. Therefore, we propose that these previous results should be reinterpreted in the framework of multiple interacting reference frames. The differences in the contributions of each reference frame with respect to our results might be due to some fundamental methodological differences, such as the use of alphanumeric characters with their intrinsic canonical orientation, the size of the explored stimuli, and the active versus passive exploration mode.

A similar interaction of reference frames was also observed in visual mental rotation tasks (Corballis et al., 1976, 1978; McMullen \& Jolicoeur, 1992). The pattern of response times excluded the involvement of a single reference frame and promoted the idea of differently contributing frames of reference. However, in contrast to the haptic mental rotation task where the egocentric reference frame predominated, the allocentric, gravitationally aligned, reference frame was found to be the heavier weighted reference frame. Interestingly, we can presume that in vision humans are better adapted to compensate for changes in their own viewing position relative to objects than for changes in the position of objects relative to themselves, whereas in haptics the opposite is true.

Since the use of multiple reference frames is in agreement with the previous findings on the mechanisms that govern haptic spatial processing, it is of interest to address this subject thoroughly. As we mentioned in the Introduction, multiple reference frames in haptics have been investigated with a task in which participants were required to orient an object to perceive it as parallel to another one. The common outcome was that the systematic deviations from parallelity were biased in the direction of the handcentered reference frame (Kappers, 2007; Volcic \& Kappers, 2008). A minor influence of the body-centered reference frame was also shown in Kappers and Viergever (2006), where deviations from veridicality were observed also in the condition in which the two hands were aligned. In general, the predominant biasing effect of the hand could differ across participants, but more importantly, it was modulated by the specific task demands. For instance, providing non-informative visual information (i.e., vision of the task workspace, but without any visual input that was directly relevant to the task) diminished the biasing influence of the hand-centered reference frame (Newport, Rabb, \& Jackson, 2002; Volcic, van Rheede, Postma, \& Kappers, 2008; Zuidhoek, Visser, Bredero, \& Postma, 2004). The visual information about the surrounding environment seemed to facilitate the contribution of the allocentric reference frame. Similarly, presenting participants with a 10-s delay between the perception of the first object and the parallel setting of the second object led to a decrease in the biasing effect (Zuidhoek, Kappers, van der Lubbe, \& Postma, 2003). This improvement was interpreted as reflecting a shift from an egocentric towards a more allocentric spatial representation over delay time, similar to the findings of several visuomotor studies (Bridgeman, Peery, \& Anand, 1997; Carrozzo, Stratta, McIntyre, \& Lacquaniti, 2002; Milner, Paulignan, Dijkerman, Michel, \& Jeannerod, 1999; Rossetti, Gaunet, \& Thinus-Blanc, 1996). These observations suggested a sort of continuum between allocentric and egocentric representations in spatial encoding, in which the available information and the task demands induce a certain ratio between the contributions of the different reference frames. In the basic case, the weight of the biasing effect of the hand-centered reference frame was estimated to be approximately .25 on average (Kappers, 2007). The biasing effect was then reduced by additional non-informative visual information or additional processing due to temporal delay. On the other hand, in the present study the weight of the hand-centered reference frame was estimated to be .62 on average. A fundamental question thus emerges: why is there such a substantial difference in the magnitude of the hand-centered reference frame contribution between the previous and the present studies? First of all, whereas the solu- tion of the parallelity task is based on an allocentric description, in the mental rotation task the use of an allocentric reference frame is not necessary per se. The concept of parallelity is implicitly defined with respect to an allocentric reference frame. Therefore, the perceiver is at least forced to strive to encode the spatial information in this reference frame. On the contrary, in the mental rotation task, the perceiver has the freedom to choose among reference frames. Second, the two tasks largely differ in their imposed temporal constraints: in the parallelity task participants took several seconds to set the object to be perceptually parallel, whereas in the mental rotation task the average response time was about $1 \mathrm{~s}$. The contribution of the hand-centered egocentric reference frame in the mental rotation task was found to be comparatively larger than in the parallelity task, and even larger than in the delayed parallelity task. In view of this comparison between studies, we could therefore speculate that as time progresses, haptic spatial processing is less influenced by egocentric reference frames and more by the allocentric one. Initially, spatial encoding might be exclusively hand-centered, then progressively more body-centered, and finally allocentric. Furthermore, the inter-participant differences between the weightings of the involved reference frames were considerably less pronounced in the mental rotation task than in the parallelity task studies. We might thus suppose that the relative contributions of reference frames are more constrained at the start of spatial processing resulting in a higher similarity across participants and, as time passes, these relative contributions change with different participant-dependent temporal evolutions.

Importantly, the framework of multiple interacting reference frames is not limited to haptic spatial processing, but it is considered to be a general principle in the way the brain transforms, combines and compares spatial representations (Carrozzo \& Lacquaniti, 1994; Carrozzo et al., 2002; Cohen \& Andersen, 2002; Pouget \& Sejnowski, 1997; Salinas \& Thier, 2000; Soechting \& Flanders, 1992, 1993). Increasing evidence suggests that spatial information can be simultaneously available in different reference frames and the appropriate information may be read out according to the ongoing task requirements. The origin of this information can be thus visual, sensorimotor, haptic or auditory and it can be coded in several reference frames, within or between modalities, which have to be combined to achieve the goals of an active perceiver.

In conclusion, we showed that in haptic mental rotation the phase shifts of the response time function are influenced by a specific integration of reference frames and are not dependent on the exclusive use of a single frame of reference. The allocentric, the hand-centered and the body-centered reference frames are the most likely reference frames involved in haptic spatial processing. Among them, the hand-centered reference frame proved to be most influential. Our results are in agreement with accumulating evidence suggesting that the combination of multiple reference frames is a mechanism governing not only haptic spatial processing, but also spatial processing in general.

\section{Acknowledgment}

This research was supported by the Netherlands Organization of Scientific Research (NWO).

\section{References}

Bridgeman, B., Peery, S., \& Anand, S. (1997). Interaction of cognitive and sensorimotor maps of visual space. Perception and Psychophysics, 59, 456-469. Carpenter, P. A., \& Eisenberg, P. (1978). Mental rotation and the frame of reference in blind and sighted individuals. Perception and Psychophysics, 23, 117-124.

Carrozzo, M., \& Lacquaniti, F. (1994). A hybrid frame of reference for visuo-manual coordination. NeuroReport, 5, 453-456. 
Carrozzo, M., Stratta, F., McIntyre, J., \& Lacquaniti, F. (2002). Cognitive allocentric representations of visual space shape pointing errors. Experimental Brain Research, 147, 426-436.

Cohen, Y. E., \& Andersen, R. A. (2002). A common reference frame for movement plans in the posterior parietal cortex. Nature Review Neuroscience, 3, 553-562.

Corballis, M. C., Nagourney, B. A., Shetzer, L. I., \& Stefanatos, G. (1978). Mental rotation under head tilt: Factors influencing the location of the subjective reference frame. Perception and Psychophysics, 24, 263-273.

Corballis, M. C., Zbrodoff, J., \& Roldan, C. E. (1976). What's up in mental rotation? Perception and Psychophysics, 19, 525-530.

Corcoran, D. W. J. (1977). The phenomena of the disembodied eye or is it a matter of personal geography? Perception, 6, 247-253.

Duke, J. D. (1966). Perception of finger drawings upon the body surface. Journal of General Psychology, 75, 305-314.

Kappers, A. M. L. (1999). Large systematic deviations in the haptic perception of parallelity. Perception, 28, 1001-1012.

Kappers, A. M. L. (2004). The contributions of egocentric and allocentric reference frames in haptic spatial tasks. Acta Psychologica, 117, 333-340.

Kappers, A. M. L. (2005). Intermediate frames of reference in haptically perceived parallelity. In A. Bicchi \& M. Bergamasco (Eds.), World haptics conference (WHC 2005): First joint eurohaptics conference and symposium on haptic interfaces for virtual environment and teleoperator systems (pp. 3-11). Los Alamitos, CA: IEEE Computer Society.

Kappers, A. M. L. (2007). Haptic space processing: Allocentric and egocentric reference frames. Canadian Journal of Experimental Psychology, 61, 208-218.

Kappers, A. M. L., \& Koenderink, J. J. (1999). Haptic perception of spatial relations. Perception, 28, 781-795.

Kappers, A. M. L., \& Viergever, R. F. (2006). Hand orientation is insufficiently compensated for in haptic spatial perception. Experimental Brain Research, 173, 407-414.

Krech, D., \& Crutchfield, R. S. (1958). Elements of psychology. New York: Knopf.

McMullen, P. A., \& Jolicoeur, P. (1992). Reference frame and effects of orientation on finding the tops of rotated objects. Journal of Experimental Psychology: Human Perception and Performance, 18, 807-820.

Milner, A. D., Paulignan, Y., Dijkerman, H. C., Michel, F., \& Jeannerod, M. (1999). A paradoxical improvement of misreaching in optic ataxia: new evidence for two separate neural systems for visual localization. Proceedings of the Royal Society of London B, 266, 2225-2229.

Natsoulas, T., \& Dubanoski, R. A. (1964). Inferring the locus and orientation of the perceiver from responses to stimulation of the skin. American Journal of Psychology, 77, 281-285.

Newport, R., Rabb, R., \& Jackson, S. R. (2002). Noninformative vision improves haptic spatial perception. Current Biology, 12, 1661-1664.
Oldfield, S. R., \& Philips, J. R. (1983). The spatial characteristics of tactile form perception. Perception, 12, 615-626.

Parsons, L. M., \& Shimojo, S. (1987). Perceived spatial organization of cutaneous patterns on surfaces of the human body in various positions. Journal of Experimental Psychology: Human Perception and Performance, 13, 488-504.

Postma, A., Zuidhoek, S., Noordzij, M. L., \& Kappers, A. M. L. (2008). Keep an eye on your hands: On the role of visual mechanisms in processing of haptic space. Cognitive Processing, 9, 63-68.

Pouget, A., \& Sejnowski, T. J. (1997). Spatial transformations in the parietal cortex using basis functions. Journal of Cognitive Neuroscience, 9, 222-237.

Prather, S. C., \& Sathian, K. (2002). Mental rotation of tactile stimuli. Cognitive Brain Research, 14, 91-98.

Rossetti, Y., Gaunet, F., \& Thinus-Blanc, C. (1996). Early visual experience affects memorization and spatial representation of proprioceptive targets. NeuroReport, 7, 1219-1223.

Salinas, E., \& Thier, P. (2000). Gain modulation: A major computational principle of the central nervous system. Neuron, 27, 15-21.

Shepard, R. N., \& Metzler, J. (1971). Mental rotation of three-dimensional objects. Science, 171, 701-703.

Soechting, J. F., \& Flanders, M. (1992). Moving in three-dimensional space: Frames of reference, vectors, and coordinate systems. Annual Review of Neuroscience, 15, $167-191$.

Soechting, J. F., \& Flanders, M. (1993). Parallel, interdependent channels for location and orientation in sensorimotor transformations for reaching and grasping. Journal of Neurophysiology, 70, 1137-1150.

Volcic, R., \& Kappers, A. M. L. (2008). Allocentric and egocentric reference frames in the processing of three-dimensional haptic space. Experimental Brain Research, $188,199-213$.

Volcic, R., Kappers, A. M. L., \& Koenderink, J. J. (2007). Haptic parallelity perception on the frontoparallel plane: The involvement of reference frames. Perception and Psychophysics, 69, 276-286.

Volcic, R., van Rheede, J. J., Postma, A., \& Kappers, A. M. L. (2008). Differential effects of non-informative vision and visual interference on haptic spatial processing. Experimental Brain Research, 190, 31-41.

Wagenmakers, E.-J., \& Farrell, S. (2004). AIC model selection using Akaike weights. Psychonomic Bulletin and Review, 11, 192-196.

Zuidhoek, S., Kappers, A. M. L., van der Lubbe, R. H. J., \& Postma, A. (2003). Delay improves performance on a haptic spatial matching task. Experimental Brain Research, 149, 320-330.

Zuidhoek, S., Visser, A., Bredero, M. E., \& Postma, A. (2004). Multisensory integration mechanisms in haptic space perception. Experimental Brain Research, 157, 265-268. 